\title{
Mechanism of Action of Xiaoyao San in Treatment of Ischemic Stroke is Related to Anti-Apoptosis and Activation of PI3K/Akt Pathway
}

This article was published in the following Dove Press journal: Drug Design, Development and Therapy

\author{
Yue $\mathrm{Xu}{ }^{1}, *$ \\ Weiyin Chen ${ }^{1}, *$ \\ Zeran Chen ${ }^{2}$ \\ Mengyuan Huang' \\ Fang Yang (D' \\ Yang Zhang' \\ 'Hospital of Chengdu University of \\ Traditional Chinese Medicine, Chengdu, \\ 6I0072, People's Republic of China; \\ ${ }^{2}$ School of Medical and Life Sciences, \\ Chengdu University of Traditional \\ Chinese Medicine, Chengdu Sichuan, \\ 6I004I, People's Republic of China \\ *These authors contributed equally to \\ this work
}

Objective: The traditional Chinese medicine (TCM) formulation Xiaoyao San (XYS) has a good clinical effect in treating ischemic stroke (IS). We explored the mechanism and material basis of XYS in IS treatment.

Methods: Network pharmacology was used to construct a network of XYS components and IS targets. R software was used to analyze the biological process and pathway analysis of the targets of XYS in IS treatment. In vitro, a model of apoptosis of PC12 cells induced by oxygen-glucose deprivation/reperfusion (OGD/R) was established to evaluate the neuroprotective effect of XYS and its influence on the expression of apoptotic protein-related genes. The affinity between the potentially active compounds in XYS and apoptotic proteins was evaluated by molecular docking.

Results: XYS was shown to have 136 chemical components that exert potential anti-IS activity by acting on 175 proteins. Bioinformatics analysis showed that apoptosis and the phosphoinositide 3-kinase/protein kinase B (PI3K-Akt) signaling pathway were the main signaling pathways of XYS. In vitro experiments showed that XYS could improve the effect of $\mathrm{OGD} / \mathrm{R}$ on PC12-cell activity $\left(\mathrm{EC}_{50}=0.43 \mathrm{mg} / \mathrm{mL}\right)$ and inhibit apoptosis. The main mechanisms were related to the improvement of oxidative stress and regulation of apoptosisrelated gene expression. Molecular docking showed that $\mathrm{C} 22, \mathrm{C} 102$ and other components in XYS had a strong affinity with apoptosis-related proteins.

Conclusion: Network pharmacology, in vitro experiments, and molecular docking were used, for the first time, to study the material basis and molecular mechanism of XYS in IS treatment from the perspective of multiple targets and multiple pathways. We provided a new approach for the future study of TCM formulations in the treatment of complex diseases.

Keywords: apoptosis, neuroprotection, biological function, pharmacological mechanism
Correspondence: Fang Yang

Hospital of Chengdu University of

Traditional Chinese Medicine, No. 39 Shier-qiao Road, Chengdu, 610075, People's Republic of China

Tel/Fax +86 28- 8778348

Email yfgreens2013@163.com

Yang Zhang

Hospital of Chengdu University of Traditional Chinese Medicine, Chengdu, 610072, People's Republic of China

Email zhangyang@cdutcm.edu.cn

\section{Introduction}

Stoke is one of the leading causes of disability and death in humans. ${ }^{1}$ More than $70 \%$ of strokes are ischemic strokes (ISs). The main cause of ISs is the stenosis or occlusion of brain arteries, including the carotid and vertebral arteries. This disorder results in insufficient blood supply to the brain and eventual necrosis of brain tissue. $^{2,3}$ About 15 million patients worldwide suffer IS each year, which leads to the death of 5 million patients. ${ }^{4}$ IS is a complex pathophysiological process involving excitatory toxicity of neurons, inflammation, energy-metabolism disorders, apoptosis, mitochondrial injury, and oxidative stress. ${ }^{5-7}$ The initiation and interaction of these mechanisms lead, eventually, to necrosis/apoptosis of neurons, 
resulting in irreversible and sustained neurological dysfunction. ${ }^{8}$ The most efficacious treatment of ISs is timely reperfusion thrombolysis in patients to improve neurological dysfunction. However, such treatment can aggravate inflammation or neuroexcitatory toxicity, thereby resulting in cerebral ischemia-reperfusion $(\mathrm{I} / \mathrm{R})$ injury. ${ }^{9,10}$ Drugs such as tissue plasminogen activator, edaravone and brain-derived neurotrophic factor have been approved for the clinical treatment of stroke, but they have limitations. For example, tissue plasminogen activator is not applicable for all stroke patients. ${ }^{11,12}$ Medicines based on plants are important for the treatment and prevention of disease. Compounds derived from naturally available materials have been used widely in the clinical management of metabolic diseases, autoimmune diseases, and cancer. $^{13,14}$ Xiaoyao San (XYS) is a traditional Chinese medicine (TCM) formulation made up of six herbs (Glycyrrhiza uralensis Fisch, Angelica sinensis (Oliv.) Diels, Wolfiporia cocos (Schwein.) Ryvarden \& Gilb., Paeonia lactiflora Pall, Atractylodes macrocephala Koidz., Bupleurum chinense DC). XYS has a good clinical effect in IS treatment, with few adverse reactions, and is very safe to use. ${ }^{15-18}$ Pharmacological studies have shown that XYS can control inflammation, reduce neuron apoptosis, and have a significant protective effect against lipopolysaccharide-induced injury to hippocampal neurons. ${ }^{19-21}$ Nevertheless, the physical basis and molecular mechanisms of XYS that underlie its neuroprotective role in IS are not known. The neuroprotective effect of XYS was explored using network pharmacology, molecular docking, and in vitro experiments. The potential mechanism of action of XYS was revealed to provide new alternative and complementary drugs for IS treatment.

\section{Materials and Methods}

\section{Databases}

With regard to network pharmacology, the databases that we used were: Traditional Chinese Medicine Systems Pharmacology Database and Analysis Platform (TCMSP; http://lsp.nwu.edu.cn/tcmsp.php/); TCMDatebase@Taiwan database (TCMD; http://tcm.cmu. edu.tw/), PubChem Compound database (www.ncbi. nlm.nih.gov/pccompound/), Chemical Book (www.chemi calbook.com/); PharmMapper (http://lilab.ecust.edu.cn/ pharmmapper/index.php/); SwissTargetPrediction (www. swisstargetprediction.ch/); DrugBank (www.drugbank.

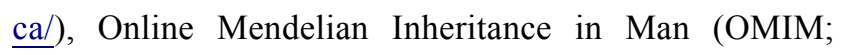

www.omim.org/), GeneCards (www.genecards.org/), DisGeNET (http://www.disgenet.org/web/disGeNET/ menu/); Treatment Targets Database (TTD; http://bidd. nus.edu.sg/); Protein Database (PDB; www.rcsb.org/), UniProt (www.rcsb.org/) and Search Tool for the Retrieval of Interacting Genes/Proteins (STRING; https://string-db.org/).

\section{Software}

We employed Discovery Studio 4.5.0 (Thermo Fisher, Boston, MA, USA), ChemDraw 15.0 (PerkinElmer, Waltham, MA, USA) and CytoScape 3.6.1 (https://cytos cape.org/) and R 3.6.3 (R Project for Statistical Computing, Vienna, Austria).

\section{Reagents}

Fetal bovine serum, phosphate-buffered saline (PBS), penicillin-streptomycin solution and Roswell Park Memorial Institute-1640 (RPMI) medium were purchased from Gibco (Grand Island, NY, USA). 4',6-diamidino2-phenylindole (DAPI) and 2',7'-Dichlorofluorescin diacetate (DCFH-DA) were obtained from US Everbright (https://www.ebgroup.cc/).

\section{Kits}

An Annexin V-fluorescein isothiocyanate/propidium iodide (FITC/PI) apoptosis detection kit was purchased from Multisciences (Lianke) Biotechnology (Hangzhou, China). Cell Counting Kit (CCK) 8 detection kit and an enhanced chemiluminescence detection (ECL) kit were obtained from Beijing 4A Biotech (Beijing, China). TRIzol ${ }^{\circledR}$ Plus RNA Purification kit and MultiScribe RT kit were purchased from Invitrogen (Carlsbad, CA, USA). A SYBR Green RT-PCR kit (QPK-201) was from Toyobo (Tokyo, Japan). The primers used in the real-time reverse transcription-quantitative polymerase chain reaction (RT-qPCR) were provided by Shengon Biotech (Shanghai, China).

\section{Antibodies}

Primary antibodies against B-cell lymphoma (Bcl)-2, Bax, phosphoinositide 3-kinase (PI3K), protein kinase B (Akt), phosphorylated (p)-PI3K, p-Akt, c-caspase 3, c-caspase 9, and $\beta$-actin were acquired from Boster Biological (Wuhan, China). Primary antibodies against immunoglobulin G-horseradish peroxidase were obtained from Beijing Bioss Biotechnology (Beijing, China). 


\section{Establishment of a Database of the Small-Molecule Compounds within XYS}

The compounds in six herbs in XYS were collected by searching TCMSP and TCMD. The missing compounds were supplemented by literature searches to ensure comprehensive and complete retrieval. Oral bioavailability (OB) and drug-likeness (DL) were used to select these compounds. Compounds with $\mathrm{OB} \geq 30 \%$ and $\mathrm{DL} \geq 18 \%$ were considered to have "good" medicinal and quasi-medicinal properties, and could be used as the potentially active components of XYS against IS. The structural formulae of potentially active compounds were searched and downloaded in PubChem, Chemicalbook and other databases, or drawn with ChemDraw. All files were saved in MOL format.

\section{Establishment of a Database for XYS-is Targets}

The structural formulae of potentially active compounds in XYS that we obtained were uploaded to PharmMapper. The targets of these compounds were obtained by reverse docking. SwissTargetPrediction was used to supplement the targets of the compounds. All docking results were combined, and repeated targets were deleted to obtain a database for XYS targets. The protein targets related to IS treatment were searched in OMIM, GeneCards, DisGeNET, TTD, and DrugBank using the keywords "IS", "ischemic stroke", "stroke" and "cerebral ischemic stroke". Simultaneously, the targets of IS were supplemented by combining literature retrieval and data mining. All retrieval results were combined and repeated targets were deleted to obtain the target database for IS.

Upon intersection of the databases for XYS targets and IS, the obtained targets were deemed to be the overlapping genes of XYS-IS. The UniProt was used to determine the standard names of these overlapping genes.

\section{Creation of an XYS-is Interaction Network}

With overlapping genes as the bond, pairs of active component-targets were constructed. Then, these pairs were imported into CytoScape to construct an XYS-IS interaction network. In this network, the nodes represent herb components and target proteins; the edges represent the interaction between herb components and target genes. The "network analyzer" plugin was used to analyze the network structure, calculate network parameters (eg, degree and betweenness centrality) to analyze preliminarily the main active components in XYS and their main targets.

\section{Creation of Protein-Protein Interaction (PPI) Networks}

STRING is an open-source database that can analyze and predict protein interactions. Overlapping genes were uploaded to STRING, and the "organism" was set as "human source". The interaction relationship between these overlapping genes was analyzed. The interaction relationship between data pairs was imported into CytoScape to construct a PPI network. In the network graph, the nodes represent target genes, and the edges represent the direct interaction between these target genes. Simultaneously, the CytohHubba plugin was used to analyze the key genes in the PPI network. These key genes were considered to be the key genes in the anti-IS action of XYS.

\section{Analyses of Pathways}

$\mathrm{R}$ was used for analyses of pathway enrichment of overlapping genes using the Gene Ontology (GO) and Kyoto Encyclopedia of Genes and Genomes (KEGG) databases. The corresponding histograms and "bubble diagrams" were drawn. Analyses of pathway enrichment involve three categories: biological process (BP), molecular function (MF) and cellular component (CC).

\section{Molecular Docking}

Molecular docking is used in computer-aided drug design. In recent years, molecular docking has been used widely in TCM research. It also provides a new strategy for the study of the material basis and mechanism of action of components within TCM formulations. ${ }^{22}$ We downloaded the appropriate crystal structures of the proteins in caspase-3, caspase-9, Bax and Bcl-2 from PDB, and imported them into Discovery Studio. Simultaneously, the XYS-IS interaction network was analyzed in depth. Compounds with a high degree of interaction were taken to be the potential active ingredients of XYS in IS treatment. The MOL files of these active ingredients were imported into Discovery Studio. The "ligand docking" module was used for molecular docking. The docking score of the target protein and ligand was taken to be the threshold value.

\section{Experiment in vitro}

Preparation of XYS Extract

The six herbs of XYS were supplied by the pharmacy department of the hospital of Chengdu University of 
TCM (Chengdu, China). These herbs were identified by Professor Weiyin Chen (Hospital of Chengdu University of TCM). Herbal specimens were kept in the standard library of Chengdu University of TCM (number: 20191123-XYS). The six herbs (G. uralensis (15 g), A. sinensis (30 g), W. cocos (30 g), P. lactiflora $(30 \mathrm{~g})$, A. macrocephala (30 g), B. chinense (30 g)) were soaked in $800 \mathrm{~mL}$ of distilled water for $30 \mathrm{~min}$. The distilled water was allowed to boil, and then allowed to simmer for 30 min. The residue was filtered and then removed. The liquid was collected, and then freeze-dried to obtain a water extract of XYS. The latter was ground into powder and stored in a dryer for subsequent use.

\section{Cell Culture}

PC12 cells were purchased from Beijing Beina Chuanglian Biotechnology Research Institute (Beijing, China). Cells were cultured in RPMI-1640 buffer supplemented with $10 \%$ fetal bovine serum and $1 \%$ penicillin-streptomycin solution at $37^{\circ} \mathrm{C}$ in a humidified environment with $5 \% \mathrm{CO}_{2}$.

\section{Oxygen-Glucose Deprivation/Reperfusion (OGD/R) Cell Model and Cell-Viability Assay}

An $\mathrm{OGD} / \mathrm{R}$ cell model was constructed in vitro to mimic I/R. Briefly, PC12 cells at the logarithmic growth stage were seeded in a 96 -well plate $\left(1 \times 10^{4} /\right.$ well $)$ and incubated in a $5 \% \mathrm{CO}_{2}$ incubator for $12 \mathrm{~h}$. After cells had attached completely to the wall, they were divided randomly into eight groups: normal; model; XYS (six doses). In addition to the normal group, the cells of the other groups were placed in a three-gas incubator $(95 \%$ $\mathrm{N}_{2}, 5 \% \mathrm{CO}_{2}, 0.2 \% \mathrm{O}_{2}$ ), cultured with glucose-free Earle's balanced salt solution, and treated with deprivation of oxygen and glucose for $3 \mathrm{~h}^{23}$ After that, the normal culture medium was replaced, and cells were placed in a $5 \% \mathrm{CO}_{2}$ incubator at $37^{\circ} \mathrm{C}$ for an additional $12,24,48$, or $72 \mathrm{~h}$. Simultaneously with the change of the normal medium, cells in the XYS groups were given different concentrations of XYS $(0.1-3.2 \mathrm{mg} / \mathrm{mL})$. After treatment, cell activity in each group at different intervention periods was measured using the CCK8 detection kit according to manufacturer instructions.

\section{Measurement of Oxidative Stress in Cells}

DCFH-DA was used as an intracellular probe to measure the ROS level in cells. Briefly, at the end of the intervention, cells were washed thrice with PBS and incubated in the dark for 20 min with $5 \mu \mathrm{L}$ of DCFH-DA. Finally, the cells were collected, washed thrice with PBS, and the mean fluorescence intensity of cells in each group was measured by flow cytometry. In addition, according to kit instructions, changes in levels of superoxide dismutase (SOD), malondialdehyde (MDA) and catalase (CAT) in cells were detected.

\section{DAPI Staining}

PC12 cells were seeded in a six-well plate and cultured in a thermostatic incubator for $12 \mathrm{~h}$. After the cells had attached completely to the wall, cells were divided randomly into five groups (normal; model; XYS (0.40, 0.80, $1.60 \mathrm{mg} / \mathrm{mL})$ ). All cells except those in the normal group underwent OGD/R. PC12 cells in the XYS group were reoxygenated and treated with XYS at different concentrations for $24 \mathrm{~h}$. At the end of the intervention, the medium was discarded, and cells were rinsed gently with PBS thrice and fixed in $4 \%$ paraformaldehyde for $15 \mathrm{~min}$ at room temperature. Finally, cells were incubated in DAPI solution for $10 \mathrm{~min}$ in the dark. Cells were washed thrice with PBS, and then photographed using an inverted fluorescence microscope (IX71; Olympus, Tokyo, Japan).

\section{Flow Cytometry}

PC12 cells were treated with the interventions as described above. After treatment, cells were collected, washed thrice with precooled PBS, and centrifuged according to the instructions of the Annexin V-FITC/PI apoptosis detection kit. Next, cells were resuspended using $500 \mu \mathrm{L}$ of binding buffer. Then, $5 \mu \mathrm{L}$ of Annexin V-FITC and PI was added, and cells were stained for $10 \mathrm{~min}$ at room temperature. Apoptosis was detected using a CytoFLEX flow (Beckman Coulter, Fullerton, CA, USA) cytometer.

\section{RT-qPCR}

mRNA expression of caspase-3, caspase-9, Bcl-2 and Bax was measured by RT-qPCR. Briefly, cells were collected at treatment cessation. TRIzol Reagent and DNaseI were employed to isolate the total cellular RNA from PC12 cells. Complimentary (c)DNA was synthesized using the MultiScribe RT kit. Finally, cDNA was amplified according to the instructions of the SYBR Green RT-PCR kit. The primer sequences (forward and reverse, respectively) were: 5"-TGCTGATGGCAACTTCAACT-3" and 5"-ATG ATGGTTCTGATCAGCTCG-3" for Bax; 5"-GGTGGAG GAACTCTTCAGGGA-3" and 5"-GGTTCAGGTACTC AGTCATCCA-3" for Bcl-2; 5"-TGGTTCATCCAG TCGCTTTG-3" and 5"-CATTCTGTTGCCACCTTTCG -3 " for caspase-3; 5"-CGAACTAACAGGCAAGCAGC $-3 "$, and 5"-ACCTCACCAAATCCTCCAGAAC-3" for 
caspase-9; 5"-TGCCCCTCCTGATGTTGC-3" and 5"-GG CTGCTGGGAATCTGAAAA-3" for PI3K; 5"-GCCACA GGTCGCTACTATGCC-3" and 5"-CACAGCCCGAAGT CCGTTA-3" for Akt; 5"-GGGAAATCGTGCGTGA CATT-3" and 5"-GCGGCAGTGGCCATCTC-3" for $\beta$ Actin. Results are expressed according to relative quantitative analyses using the $2^{-\Delta \Delta \mathrm{CT}}$ method.

\section{Western Blotting}

After treatment, the total cell protein was extracted with RIPA cell lysates containing $1 \%$ phosphatase inhibitor and $1 \%$ protease inhibitor. The protein concentration of each sample was measured using a bicinchoninic acid (BCA) protein detection kit. After that, an appropriate amount of protein loading buffer was added to adjust the protein concentration in the sample. Then, protein was denatured by boiling water for 5-10 $\mathrm{min}$. Protein was stored at $-20^{\circ} \mathrm{C}$ for subsequent experiments. Sodium dodecyl sulfate-polyacrylamide gel electrophoresis was used to isolate protein samples $(30 \mu \mathrm{g})$. Protein was transferred to a polyvinylidene difluoride (PVDF) membrane. The latter was placed in 5\% skimmed milk and sealed for 2 $\mathrm{h}$ at room temperature. The PVDF membrane was incubated overnight at $4^{\circ} \mathrm{C}$ with primary antibodies (Bcl-2, 1: 500 dilution; Bax, 1:500; PI3K, 1:500; Akt, 1:500; p-PI3K, 1:500; p-Akt, 1: 500; c-caspase 3, 1:500; c-caspase 9, 1:500; $\beta$ actin, 1:3000). After washing the PVDF membrane thrice with Tris-buffered saline/Tween 20, it was incubated with horseradish peroxidase-conjugated secondary antibody (1:5000 dilution) for $1 \mathrm{~h}$. Then, the ECL kit was used to develop the protein. Images were recorded and, finally, relative expression of protein documented.

\section{Statistical Analyses}

All experiments were repeated and the results were expressed as the mean \pm SD value of the three experiments. SPSS 19.0 (IBM, Armonk, NY, USA) was used to analyze data. The difference between groups was analyzed by one-way analysis of variance. $P<0.05$ was considered significant.

\section{Results}

\section{Active Compounds}

According to the retrieval strategy and screening criteria, 136 potentially active compounds in XYS were collected: 92 compounds were identified in G. uralensis, two in A. sinensis, 14 in $W$. cocos, 12 in P. lactiflora, seven in A. macrocephala, and 17 in B. chinense (some of the compounds were found in several herbs). The basic information of these active compounds is shown in Table S1.

\section{Target Library}

A total of 136 potentially active compounds in XYS had 347 active target genes (Figure 1). Simultaneously, 3024 target genes related to IS were collected by database retrieval and literature searching. By taking the intersection of two target sets, 175 overlapping genes were obtained, which were the functional target genes of XYS used against IS.

\section{XYA-is Interaction Network}

There were 1041 molecule-target pairs in the XYS-IS interaction network (Figure 2A), including 136 compounds and 175 protein targets. In Figure 2A, the triangle node represents compounds, the rhombohedral node denotes herbs, and the circular node represents the protein target. Through analyses of the network, we found that the degrees of components such as C15, C49, C13, C132, $\mathrm{C} 102, \mathrm{C} 22$ and $\mathrm{C} 12$ were relatively large, indicating that these components might be the main active components in XYS. Simultaneously, several components could act on multiple targets. These data suggested that XYS could improve IS by acting upon multiple components and multiple targets (Table 1).

\section{PPI Networks}

CytoScape was used to draw a PPI network (Figure 2B). Altogether there were 175 nodes and 1847 edges in the diagram. The node size and color depth represented the degree value of the target. The larger the node and the darker the color, the larger was the degree value. The larger the degree, the more important was the part played in the PPI. The degree of interleukin-6, caspase3 , vascular endothelial growth factor- $\alpha$, mycogene, mitogen-activated protein kinase (MAPK)8, EGFR, mammalian target of rapamycin and other genes in the PPI network was $\geq 50$, suggesting that these genes were key for XYS treatment of IS (Figure 2C).

\section{Biological Function and Pathway Enrichment}

Analyses using the GO database showed that the main enrichment of genes in the BP category was "xenobiotic metabolism", "energy pathways", "signal transduction", "apoptosis", and "cell communication". The main MF categories were "protein-tyrosine kinase activity", "catalytic activity", "protein serine/threonine kinase activity", "ligand-dependent nuclear receptor 


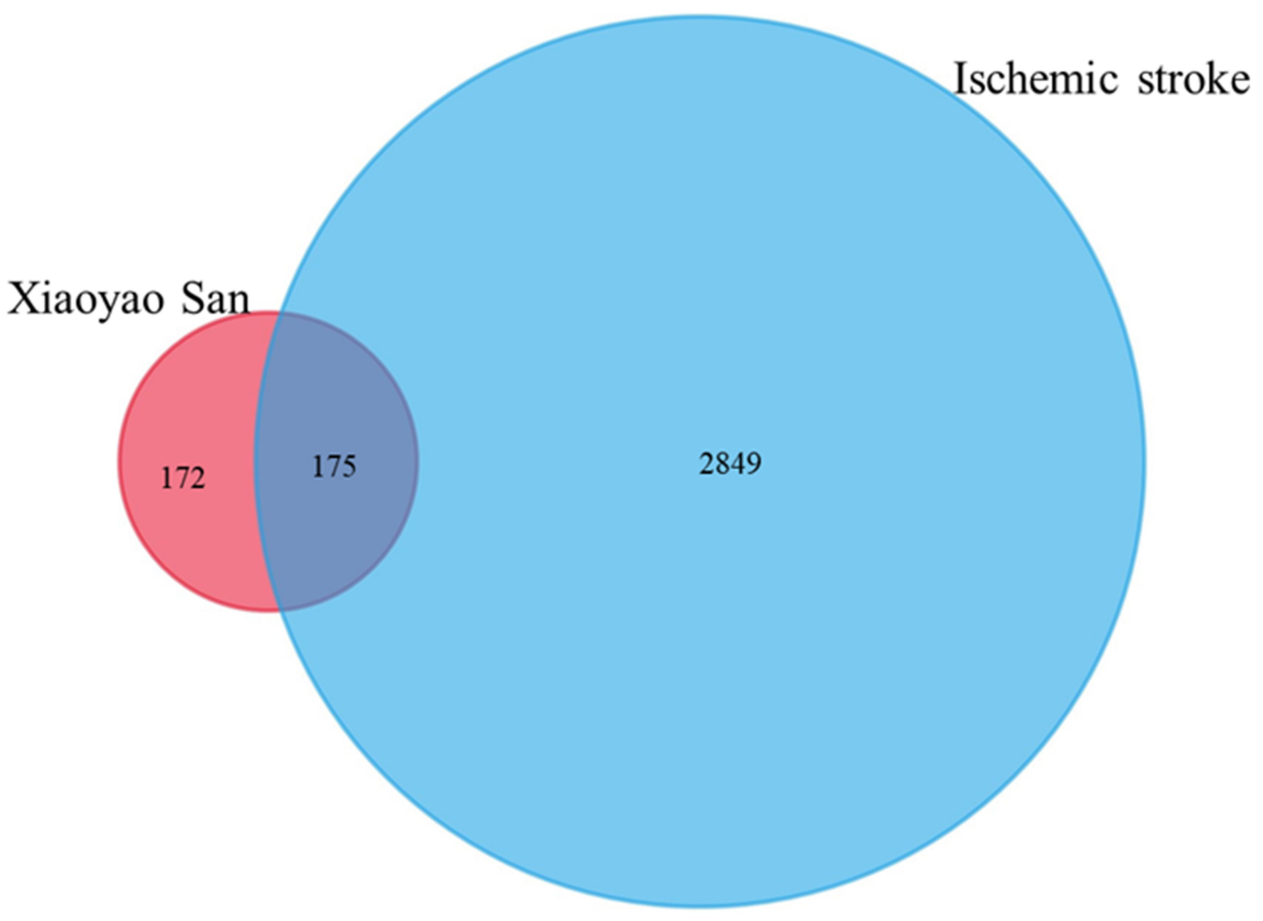

Figure I The intersection of the library of compound targets and the library of ischemic stroke (IS) targets.

A

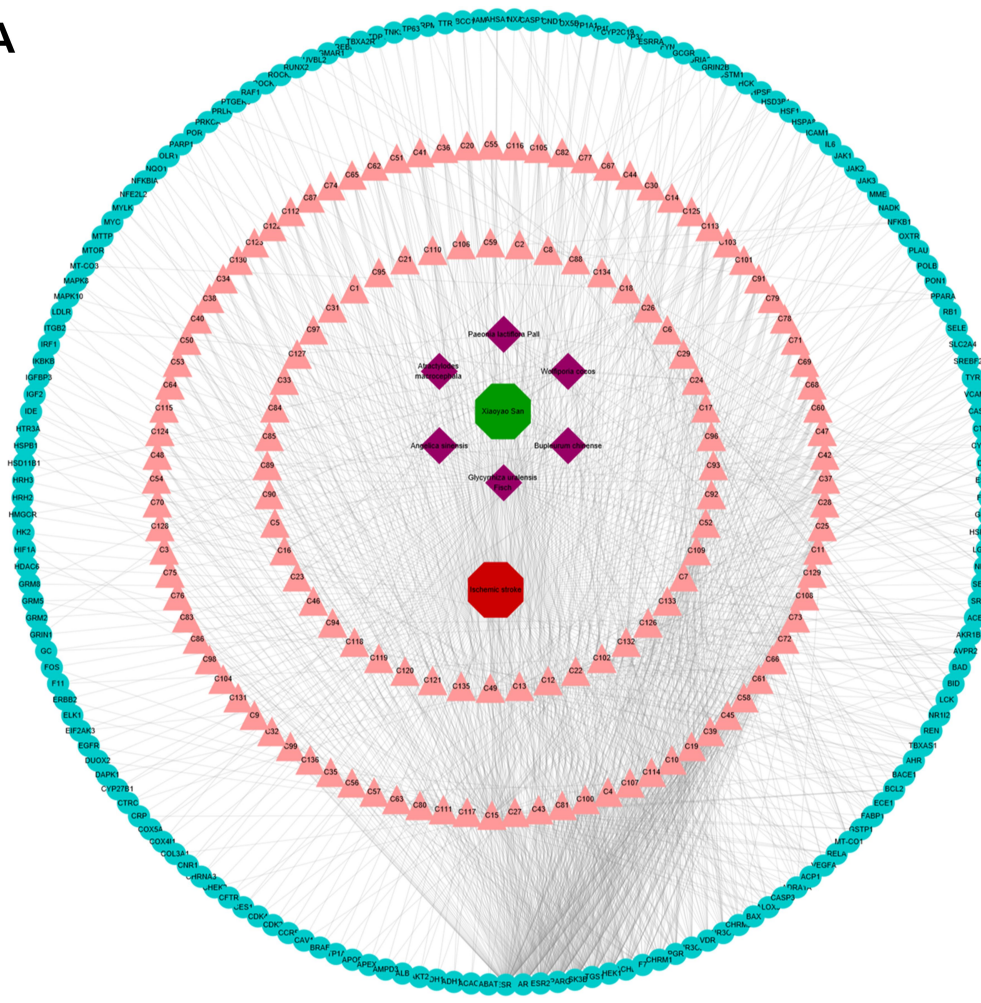

B

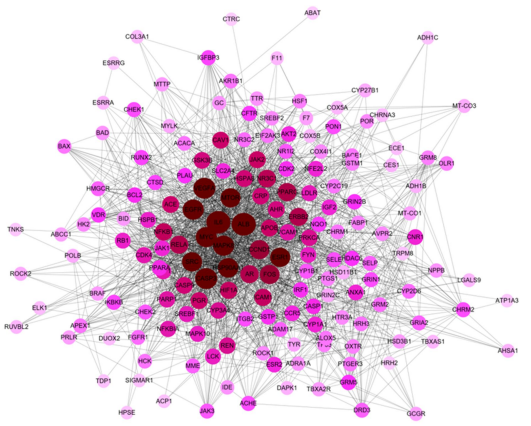

C

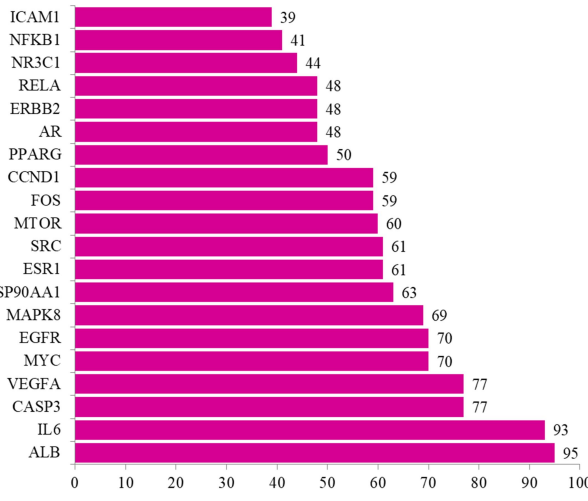

Figure 2 Networks for (A) XYS-IS interaction and (B) protein-protein interaction (PPI). (C) Hub genes of the PPI network.

activity", "kinase activity", "extracellular ligand-gated "external side of plasma membrane", "integral to ion channel activity", and "oxidoreductase activity". plasma membrane", and "mitochondrial outer memThe main CC categories were "extracellular space", brane" (Figure 3, Table S2). 
Table I Information on the Top-20 Compounds in Xiaoyao San (XYS) That Act Against Ischemic Stroke (IS)

\begin{tabular}{|c|c|c|c|c|c|c|}
\hline ID & Compound & $\begin{array}{l}\text { OB } \\
\text { (\%) }\end{array}$ & DL & Degree & $\begin{array}{l}\text { Betweenness } \\
\text { Centrality }\end{array}$ & Herb \\
\hline $\mathrm{C7}$ & Vestitol & 74.66 & 0.21 & 17 & 0.00882156 & Glycyrrhiza uralensis \\
\hline $\mathrm{Cl} 2$ & Phaseolinisoflavan & 32.01 & 0.45 & 20 & 0.01331739 & Glycyrrhiza uralensis \\
\hline $\mathrm{Cl} 3$ & Phaseol & 78.77 & 0.58 & 23 & 0.00653228 & Glycyrrhiza uralensis \\
\hline $\mathrm{Cl} 5$ & Naringenin & 59.29 & 0.21 & 60 & 0.0619301 & Glycyrrhiza uralensis \\
\hline $\mathrm{C} 22$ & Licoisoflavone B & 38.93 & 0.55 & 20 & 0.00510112 & Glycyrrhiza uralensis \\
\hline C24 & Licoisoflavanone & 52.47 & 0.54 & 12 & 0.00511129 & Glycyrrhiza uralensis \\
\hline $\mathrm{C} 27$ & Licochalcone B & 76.76 & 0.19 & 15 & 0.00774606 & Glycyrrhiza uralensis \\
\hline C29 & Licoagroisoflavone & 57.28 & 0.49 & 12 & 0.00605536 & Glycyrrhiza uralensis \\
\hline $\mathrm{C} 43$ & Glycyrrhiza flavonol A & 41.28 & 0.6 & 13 & 0.00361123 & Glycyrrhiza uralensis \\
\hline C49 & Glyasperin C & 45.56 & 0.4 & 29 & $0.0227484 \mid$ & Glycyrrhiza uralensis \\
\hline C52 & Glepidotin A & 44.72 & 0.35 & 16 & 0.00773203 & Glycyrrhiza uralensis \\
\hline C8I & $\begin{array}{l}\text { 2-(3,4-dihydroxyphenyl)-5,7-dihydroxy-6-(3-meThylbut- } \\
\text { 2-enyl)chromone }\end{array}$ & 44.15 & 0.41 & 12 & 0.00140717 & Glycyrrhiza uralensis \\
\hline $\mathrm{C} 92$ & $(-)$-Medicocarpin & 40.99 & 0.95 & 13 & 0.00477504 & Glycyrrhiza uralensis \\
\hline $\mathrm{C} 93$ & Trametenolic acid & 38.71 & 0.8 & 13 & 0.00127285 & Wolfiporia cocos \\
\hline C96 & Poricoic acid $\mathrm{A}$ & 30.61 & 0.76 & 13 & 0.00127285 & Wolfiporia cocos \\
\hline $\mathrm{ClO}$ & Cerevisterol & 37.96 & 0.77 & 20 & 0.0047771 & Wolfiporia cocos \\
\hline $\mathrm{Cl09}$ & $\alpha$-spinasterol & 42.98 & 0.76 & 17 & 0.00910366 & Bupleurum chinense \\
\hline $\mathrm{Cl} 26$ & | 4-acetyl- | 2-senecioy|-2E,8E, I0E-atractylentriol & 60.31 & 0.31 & 19 & 0.01170167 & Atractylodes macrocephala \\
\hline $\mathrm{Cl} 32$ & Benzoyl paeoniflorin & 31.27 & 0.75 & 20 & 0.0085959 & Paeonia lactiflora \\
\hline $\mathrm{Cl} 33$ & Albiflorin & 66.64 & 0.33 & 18 & 0.0117153 & Paeonia lactiflora \\
\hline
\end{tabular}

Analyses of pathway enrichment using the KEGG database showed the main pathways to be enriched were "endocrine resistance", "apoptosis", "PI3K-Akt signaling pathway", "p53 signaling pathway", "apoptosis-multiple species", and "MAPK signaling pathway". These results suggested that XYS could act against IS through multiple pathways (Figure 4, Table S3).

\section{Molecular Docking}

Analyses of molecular-docking data showed that the active compounds in XYS (C15, C13, C49, C12, C22, C102, C132) had strong binding with the proteins related to apoptosis. Hence, the mechanism of action of XYS against IS was closely related to apoptosis. The molecular docking between $\mathrm{C} 12$ and caspase-3 (3H0E), $\mathrm{C} 22$ and $\mathrm{Bcl}-2$ (6RNU), C102 and Bax (2G5B), and C102 and caspase-9 (2AR9) was strong (Figure 5). Hence, these compounds could connect to the active site of the protein.

\section{XYS Protects Against Injury to PCI2}

\section{Cells in vitro}

\section{Viability of OGD/R Model Cells is Improved}

We wished to ascertain the neuroprotective effect of YXS against cerebral $\mathrm{I} / \mathrm{R}$ injury. We used the CCK8 kit to determine the survival of $\mathrm{PC} 12$ cells after $\mathrm{OGD} / \mathrm{R}$. Compared with the normal control group, OGD/R inhibited the survival of PC12 cells significantly. Survival of PC12 cells after XYS treatment was significantly higher than that in the model group, indicating that XYS could improve the cell damage caused by OGD/R. In addition, different doses of XYS had the greatest effect of averting the death of PC12 cells at $24 \mathrm{~h}$ of intervention (Figure 6A). Therefore, in subsequent experiments, $24 \mathrm{~h}$ was the optimal duration for XYS intervention. Importantly and interestingly, the ability to save the vitality of XYS was dose-dependent (halfmaximal drug response $\left.\left(\mathrm{EC}_{50}\right)=0.4723 \mathrm{mg} / \mathrm{mL}\right)$ (Figure 6B). Due to reduced cell activity in the high-dose group (54\% cell activity at an XYS concentration of $3.2 \mathrm{mg} /$ $\mathrm{mL}$ ) (Figure 6B), 0.4, 0.8 and $1.6 \mathrm{mg} / \mathrm{mL}$ were selected as doses in subsequent experiments.

\section{XYS Improves Intracellular Oxidative Stress}

Oxidative stress is one of the important causes for the occurrence and development of IS. IS also believed to be the main cause of irreversible brain damage in IS patients. PC12 cells in the OGD/R model group showed obvious oxidative stress and produced excessive ROS (Figure 7). In the OGD/R model group, levels of the antioxidant enzymes SOD and CAT in cells were decreased significantly, and the MDA level 


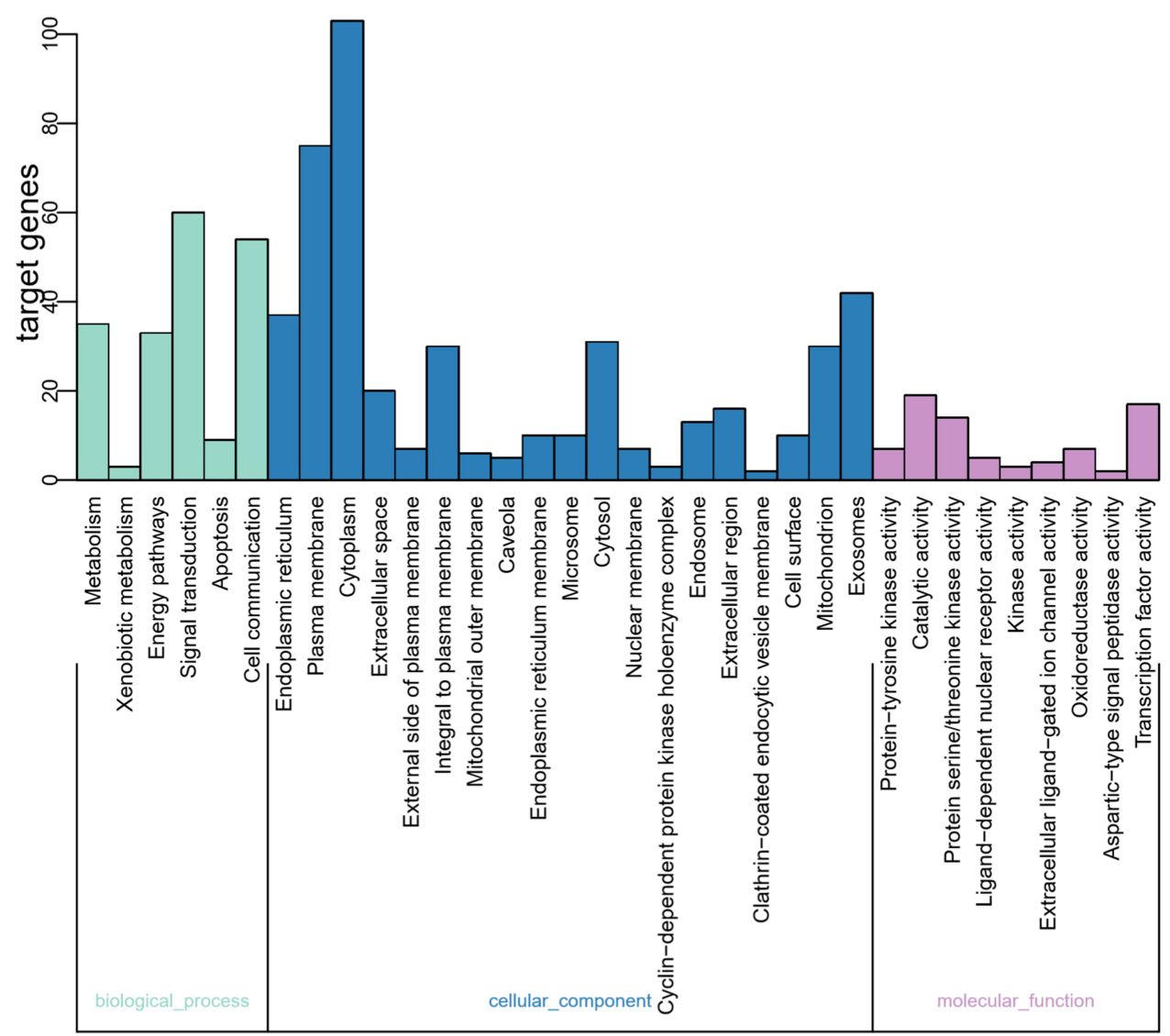

Figure 3 Analyses of functional enrichment using the GO database.

was increased significantly. After XYS treatment, the ROS level in cells decreased significantly, and levels of SOD, CAT, and MDA returned to normal. These results suggested that XYS helped to restore the redox balance in OGD/ R-treated PC12 cells.

\section{XYS Protects Neurons from the Apoptosis Induced by OGD/R}

We wished to ascertain if the effect of XYS on increasing the viability of PC12 cells was related to the inhibition of apoptosis. DAPI staining and flow cytometry were used to detect cell apoptosis. The nuclei of normal cells were intact, and weak fluorescence could be observed (Figure 8A). Cells that underwent $\mathrm{OGD} / \mathrm{R}$ showed increased fluorescence, indicating that the nuclear morphology of these cells had undergone apoptotic changes. Flow cytometry confirmed the anti-apoptotic effect of XYS. Compared with normal cells, the percentage apoptosis of $\mathrm{PC} 12$ cells treated by $\mathrm{OGD} / \mathrm{R}$ was increased, whereas XYS treatment could inhibit the apoptosis of $\mathrm{PC} 12$ cells significantly in a dose-dependent manner (Figure 8B). Taken together, these results suggested that XYS could inhibit the apoptosis of PC12 cells induced by OGD/R.

\section{XYS Regulates Expression of Apoptotic Protein-Related Genes}

We measured the expression of apoptosis-related proteins in $\mathrm{PC} 12$ cells after OGD/R. Compared with the normal control group, mRNA and protein expression of caspase-3, caspase- 9 and Bax in the OGD/R group were increased, whereas mRNA and protein expression of Bcl-2 were reduced significantly. Interestingly, the addition of XYS at different concentrations could reverse these changes, suggesting that XYS could inhibit the apoptosis of neurons under $\mathrm{I} / \mathrm{R}$ by regulating the expression of apoptosis-related genes (Figures 9 and 10).

\section{XYS Activates the PI3K/Akt Pathway}

Network pharmacology suggested that the PI3k/Akt pathway was the main mechanism of action in IS treatment by XYS. Therefore, we evaluated the effect of XYS on the mRNA and protein expression of PI3k and Akt in PC12 cells. OGD/R inhibited mRNA expression of PI3K and Akt significantly in PC12 cells (Figures 9 and 10). Compared with the normal group, although protein expression of PI3K and Akt did not change significantly, 


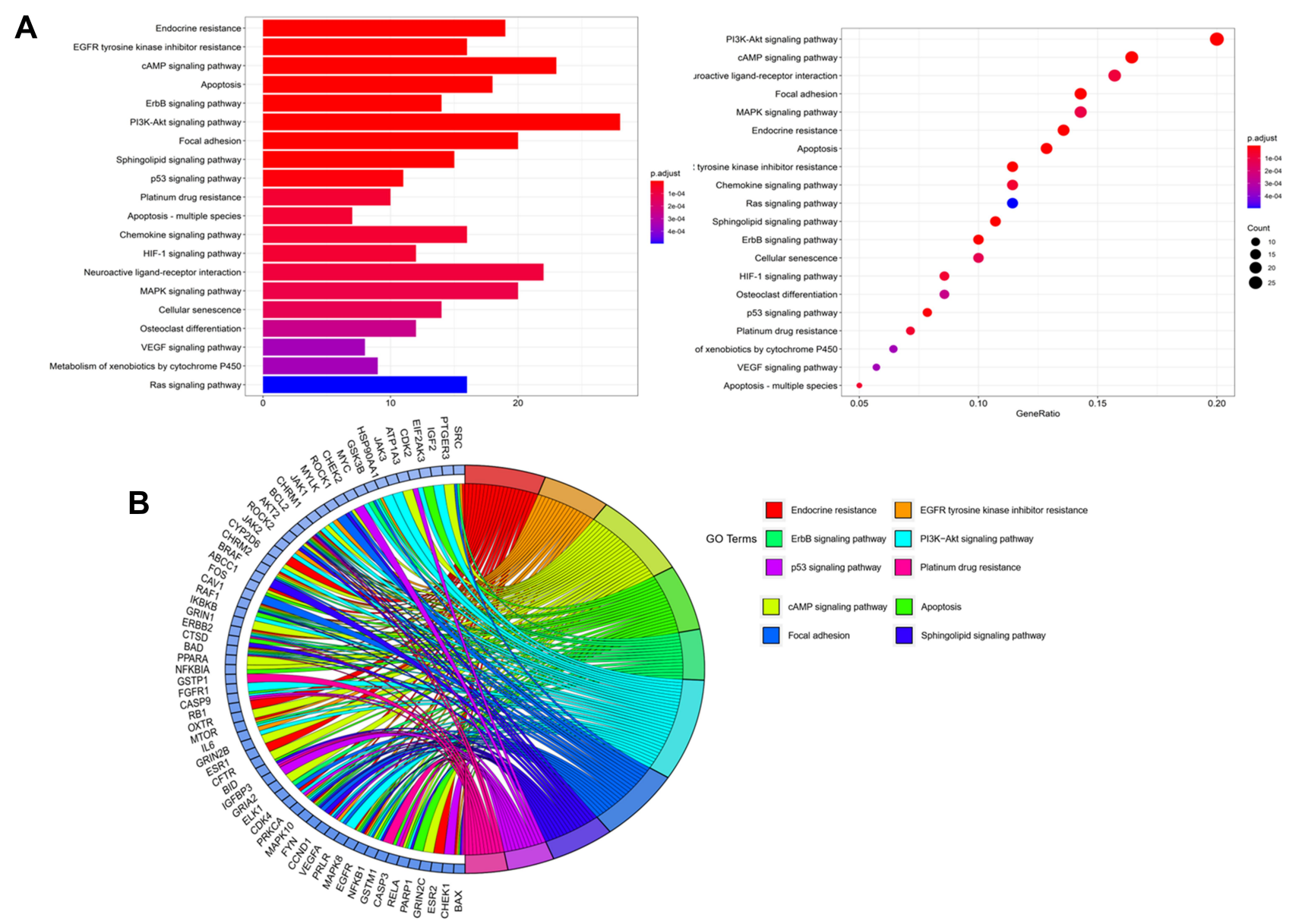

Figure 4 Analyses of pathway enrichment using the KEGG database. (A) Bar chart and bubble chart; (B) loop graph.

phosphorylation of PI3K and Akt was inhibited significantly. Fortunately, XYS therapy could reverse this inhibition. These results indicated that the PI3k/Akt pathway was involved in the anti-IS action of XYS.

\section{Discussion}

Apoptosis a normal physiological activity in cells. If apoptosis becomes abnormal, it can cause cancer, rheumatoid arthritis, atherosclerosis and stroke. Increasing numbers of studies have shown that drugs from plants can inhibit/ promote apoptosis. In other words, apoptosis is an important way for drugs from plants to play a part in the treatment and prevention of diseases. ${ }^{24-26}$ The IS eventually leads to the death of many neurons. Increasing numbers of reports have indicated that apoptosis is the main mode of neuronal cell death caused by IS. ${ }^{27,28}$ XYS is one of the main TCM formulations used for the clinical treatment of IS. Its efficacy and safety have been confirmed by several clinical trials, but the mechanism of action and material basis of IS treatment of IS are not clear. In the present study, network pharmacology, in vitro experiments, and computer-aided design were combined to comprehensively and systematically explore the mechanism of action and material basis of XYS.

In the initial network-pharmacology analyses, we found that the signaling pathways mainly involved in XYS treatment of IS were apoptosis, PI3K-Akt signaling pathway, p53 signaling pathway, and MAPK signaling pathway. Simultaneously, these pathways are closely related to apoptosis to a large extent, which suggests that the anti-IS effect of XYS is related to apoptosis, which was confirmed by subsequent in vitro experiments. The results of in vitro cell experiments showed that XYS could protect against the neuron death caused by OGD/R.

Studies have shown that reduction in transient blood flow can cause the production of many ROS, such as the superoxide anion, hydrogen peroxide, and hydroxyl radical, which leads to I/R injury. ${ }^{29}$ These transient emergencies are also thought to be one of the main causes of neuron damage caused by IS. Excessive ROS can cause oxidative stress in 


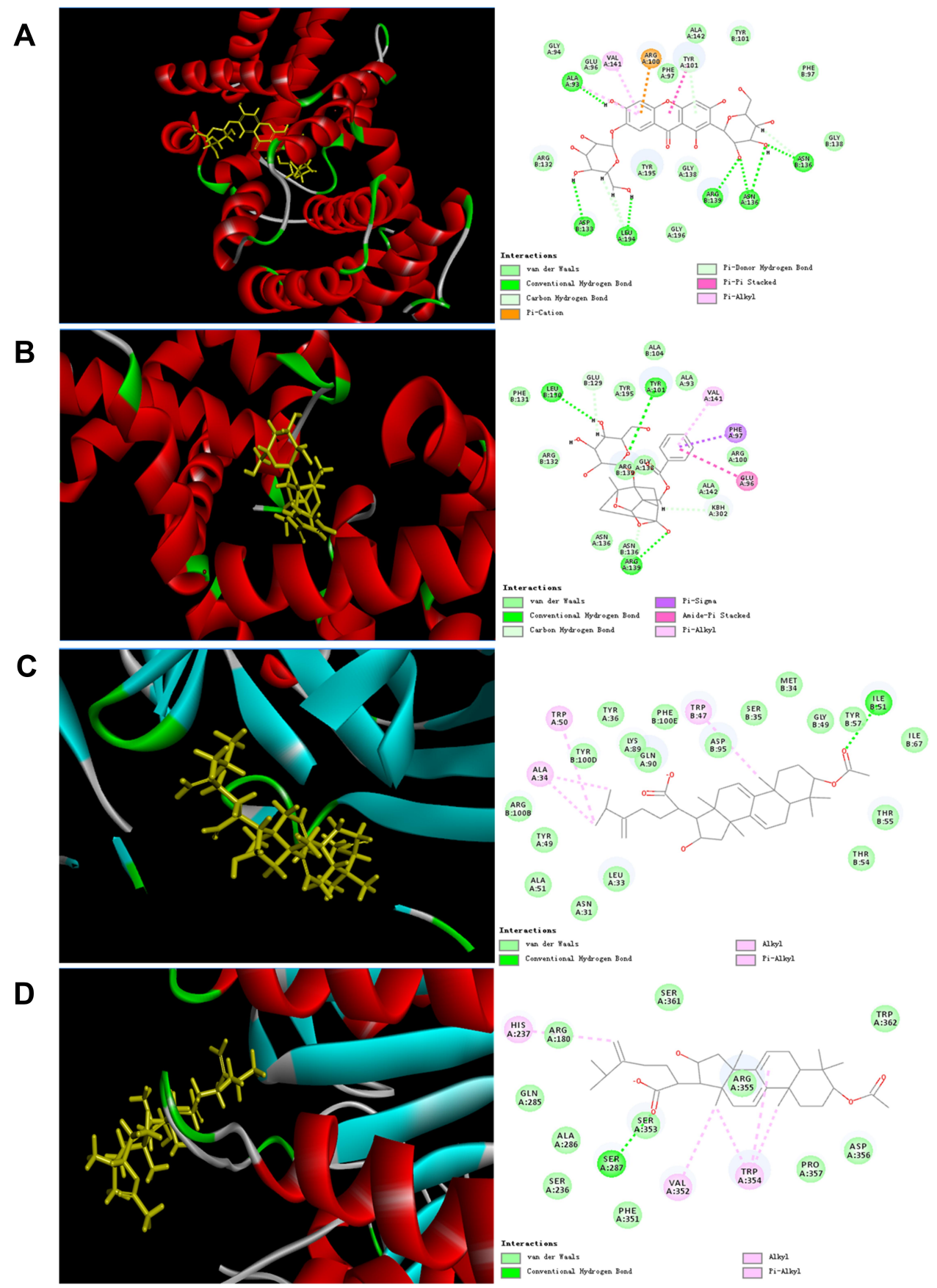

Figure 5 Partial results of molecular docking. Molecular docking of (A) Cl2 and caspase-3 (3H0E), (B) C22 and Bcl-2 (6RNU), (C) CI02 and Bax (2G5B), (D) Cl02 and caspase-9 (2AR9).

cells, resulting in oxidative damage to DNA, mitochondrial dysfunction, and abnormal expression of proteins, which lead to apoptosis. ${ }^{30}$ Therefore, targeting ROS is considered to be an effective way to protect neurons from oxidative stress-induced damage. We found that XYS could improve oxidative stress in PC12 cells and reduce ROS production. MDA levels are increased significantly under oxidative stress, which leads to cell damage. Levels of the important
ROS-scavenging enzymes SOD and CAT (which maintain the redox balance in the body) are reduced significantly, which further aggravates the damage caused by oxidative stress. Therefore, SOD and CAT are also considered to be biomarkers of oxidative stress. ${ }^{31,32}$ Interestingly, we showed that XYS could increase the level of the antioxidant enzymes SOD and CAT significantly, and inhibit MDA production, in PC12 cells. 
A

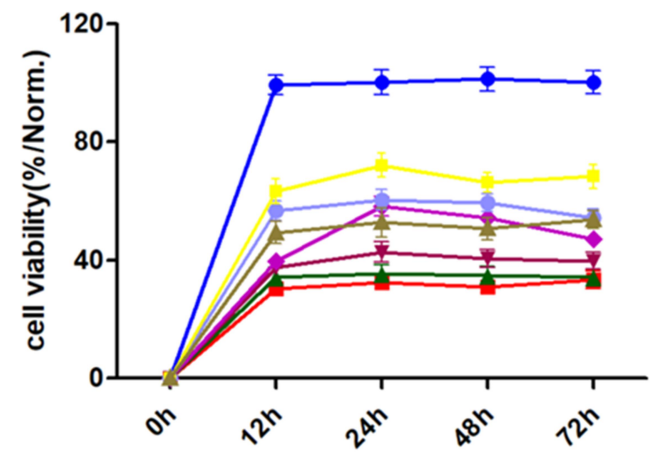

$\rightarrow$ Normal

$\rightarrow$ Model

$+0.1 \mathrm{mg} / \mathrm{mL}$

$\rightarrow 0.2 \mathrm{mg} / \mathrm{mL}$

$\rightarrow 0.4 \mathrm{mg} / \mathrm{mL}$

$\rightarrow 0.8 \mathrm{mg} / \mathrm{mL}$

$1.6 \mathrm{mg} / \mathrm{mL}$

$\pm 3.2 \mathrm{mg} / \mathrm{mL}$
B

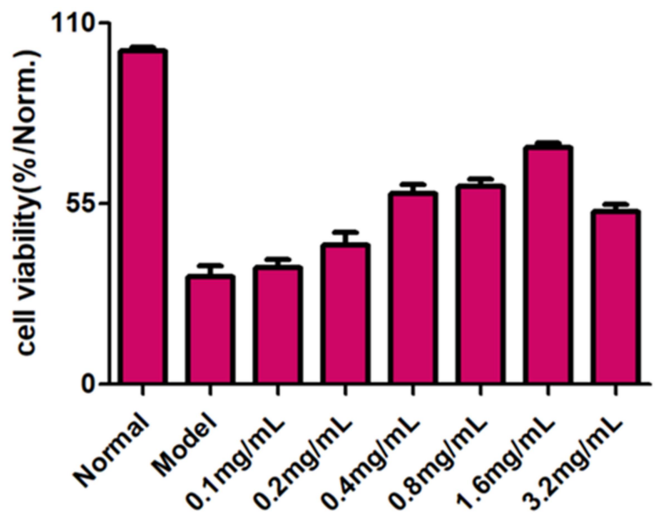

Figure 6 Cell viability. Cell viability of OGD/R-induced PCI2 cells (A) at 12, 24, 48, and $72 \mathrm{~h}$ in the presence or absence of XYS, and (B) after treatment with different concentrations of XYS for $24 \mathrm{~h}$.
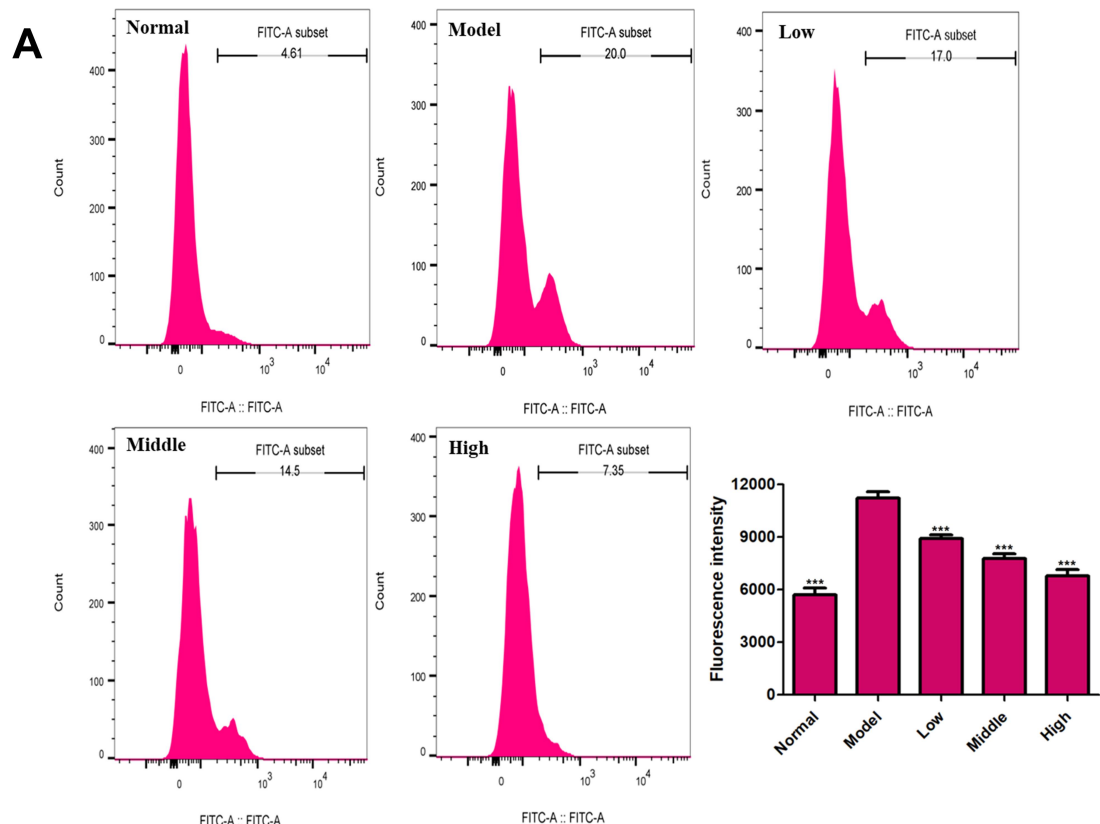

B
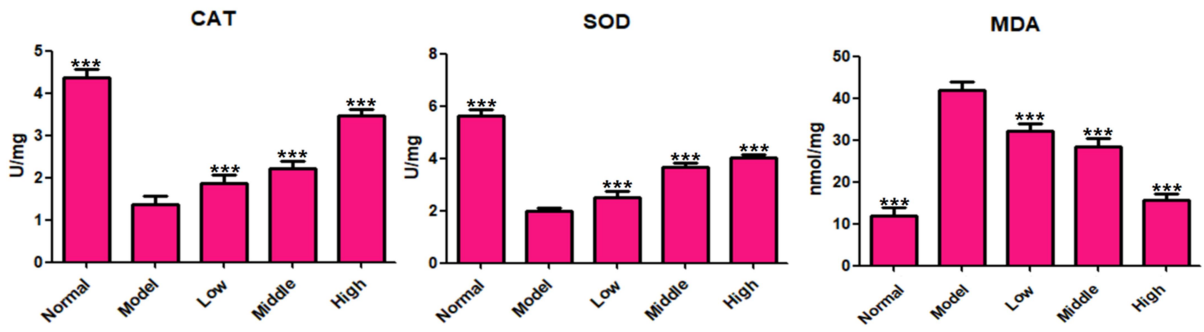

Figure 7 Effects of XYS $(0.4,0.8$ and $1.6 \mathrm{mg} / \mathrm{mL})$ on oxidative stress in PCI2 cells after 24-h treatment. (A) Flow-cytometry results for ROS. (B) Levels of CAT, SOD and MDA. Data are the mean $\pm S D(n=3)$. $* * * P<0.001$ vs the model group (OGD/R-induced PCI 2 cells without treatment with $X Y S)$.

Further studies found that the neuroprotective effect of XYS was related to anti-apoptosis, and that the mechanism was related to inhibition of apoptosis, as well as regulation of expression of caspase-3, caspase-9, Bax and Bcl-2, which verified the results of network pharmacology. The PI3K/AkT signaling pathway is involved in the proliferation, differentiation and ROSinduced apoptosis of cells, and is considered to be a potential mechanism of neuroprotection. ${ }^{33-36}$ When PI3K is phosphorylated, it catalyzes migration of the 


\section{A}

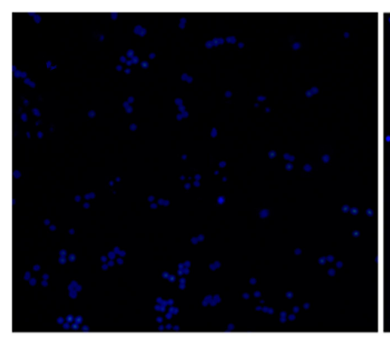

Normal

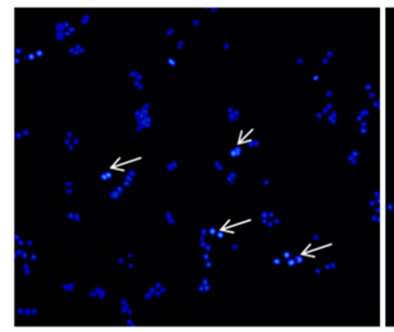

Middle
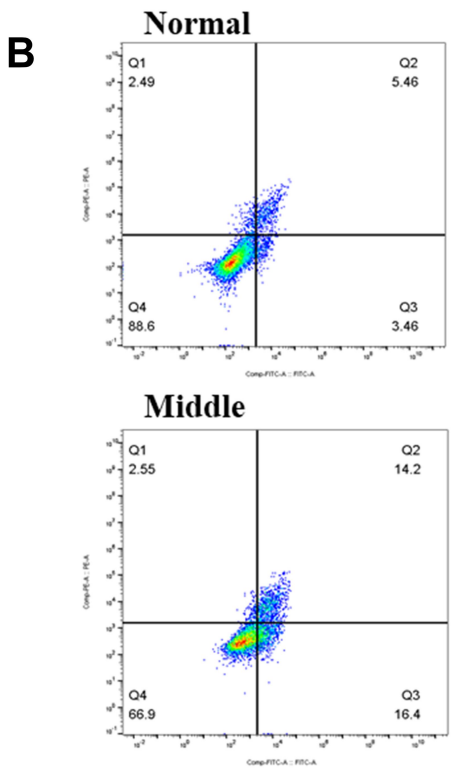

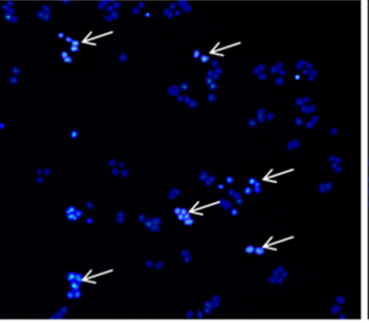

Model

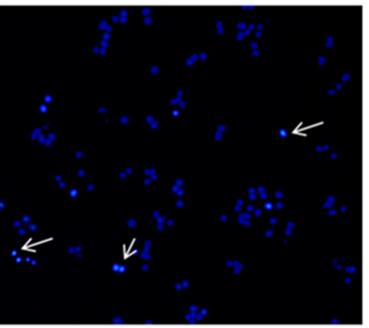

High
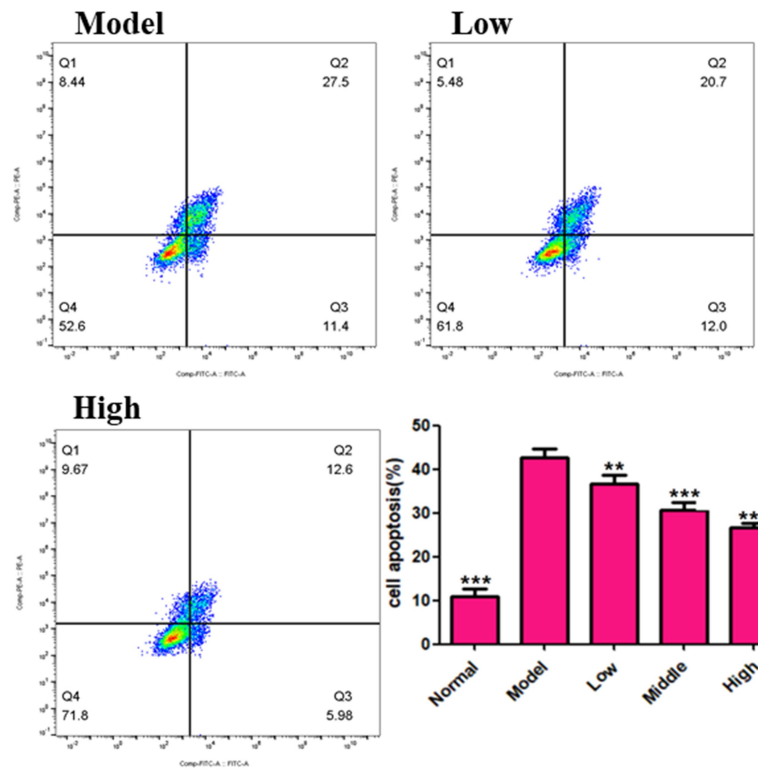

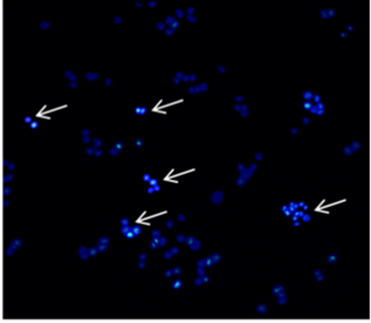

Low

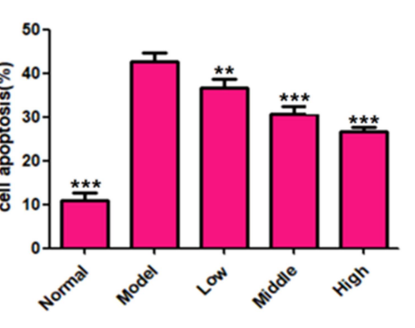

Figure 8 XYS $(0.4,0.8$ and $1.6 \mathrm{mg} / \mathrm{mL})$ protects against PCI2 injury in vitro at $24 \mathrm{~h}$. (A) DAPI staining; $(\mathbf{B})$ flow cytometry. Data are the mean \pm SD $(\mathrm{n}=3)$. The white arrow indicates that the cell is showing a significant apoptotic signal (nuclear shrinkage). $* * P<0.01$, $* * * P<0.001$ vs the model group (OGD/R-induced PCI 2 cells without treatment with $\mathrm{XYS}$ ).

downstream effector protein Akt from the cytoplasm to the membrane and activates it fully. Activated Akt can lead to increased expression of anti-apoptotic genes (eg, Bcl-2) in cells and correspondingly, reduced expression of pro-apoptotic genes (eg, Bax) and inhibition of a caspase-3- and caspase-9-mediated apoptosis cascade. ${ }^{35}$ Consistent with the predicted results of network pharmacology, XYS was found to upregulate mRNA expression of PI3K and Akt, increase phosphorylation of these proteins, and activate the PI3K-Akt signaling pathway. Moreover, the molecular-docking experiments revealed that the compounds in XYS had a strong affinity with apoptosis-related proteins, which indicated, preliminarily, that these components were the main active components of XYS.

\section{Conclusions}

Network pharmacology, in vitro experiments, and molecular docking were used, for the first time, to study the material basis and molecular mechanism of XYS in IS treatment from the perspective of multiple targets and multiple pathways. We provided a new approach for the future study of TCM formulations in the treatment of complex diseases. 

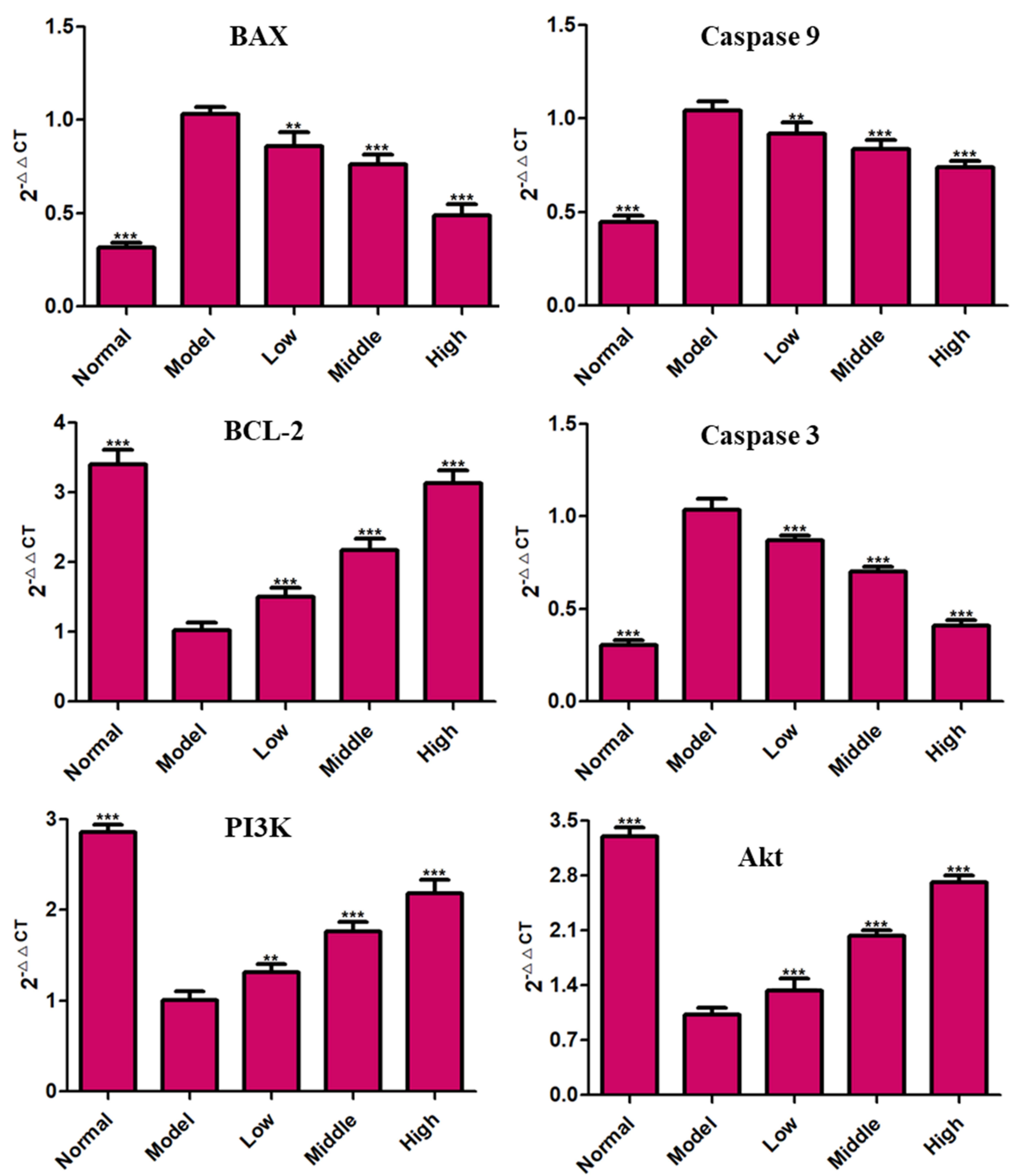

Figure 9 RT-qPCR. Cells were treated with XYS $(0.4,0.8$ or $1.6 \mathrm{mg} / \mathrm{mL})$ for $24 \mathrm{~h}$. Data are the mean \pm SD $(\mathrm{n}=3)$. **P<0.0I, ***P<0.00I vs the model group $(\mathrm{OGD} /$ $\mathrm{R}$-induced $\mathrm{PCI} 2$ cells without treatment with $\mathrm{XYS}$ ). 

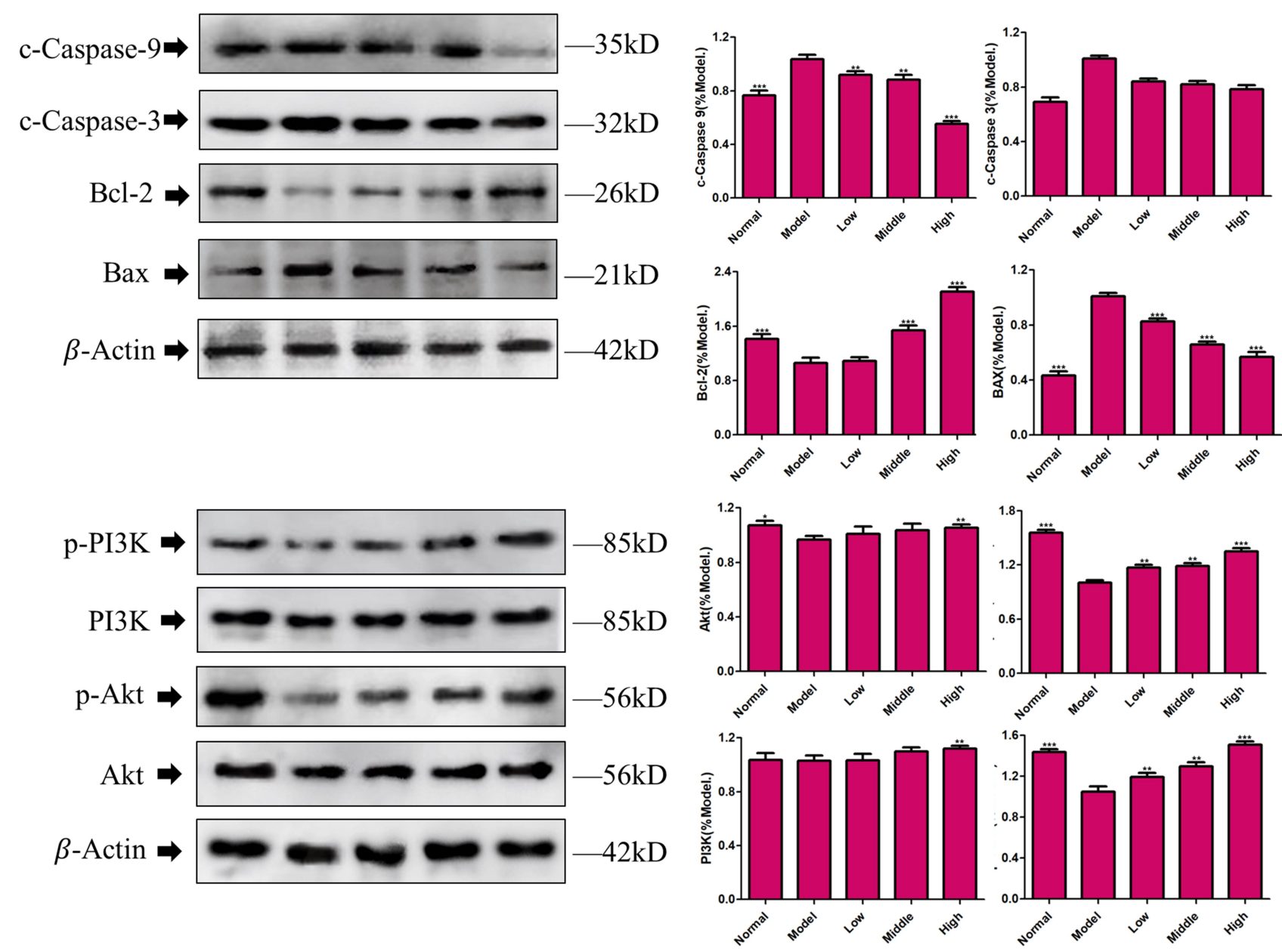

Figure 10 Western blotting. Cells were treated with XYS $(0.4,0.8$ or $1.6 \mathrm{mg} / \mathrm{mL})$ for $24 \mathrm{~h}$. Data are the mean $\pm S D(n=3)$. $* P<0.05, * * P<0.0 \mathrm{I}, * * * P<0.00 \mathrm{I}$ vs the model group (OGD/R-induced PCI2 cells without treatment with XYS).

\section{Acknowledgment}

This work was supported by the Foundation of Chengdu University of Traditional Chinese Medicine (grant number 030038084) and "Xinglin Scholar" research project of chengdu university of Traditional Chinese Medicine (grant number ZYTS2019014).

Yue $\mathrm{Xu}$ and Weiyin Chen contributed equally to this paper and should be considered as the first authors.

\section{Disclosure}

The authors declare that there is no conflict of interest.

\section{References}

1. Dabrowska-Bender M, Milewska M, Golabek A, DudaZalewska A, Staniszewska A. The impact of ischemic cerebral stroke on the quality of life of patients based on clinical, social, and psychoemotional factors. J Stroke Cerebrovasc Dis. 2017;26:101-107. doi:10.1016/j. jstrokecerebrovasdis.2016.08.036
2. Jin R, Yang G, Li G. Inflammatory mechanisms in ischemic stroke: role of inflammatory cells. J Leukoc Biol. 2010;87:779-789. doi:10.1189/jlb.1109766

3. Lakhan SE, Kirchgessner A, Hofer M. Inflammatory mechanisms in ischemic stroke: therapeutic approaches. J Transl Med. 2009;7:97. doi:10.1186/1479-5876-7-97

4. Wardlaw JM, Zoppo G, Yamaguchi T, Berge E. Acute stroke intervention: a systematic review. JAMA. 2015;14:1451-1462.

5. Lalu MM, Fergusson DA, Cheng W, et al. Identifying stroke therapeutics from preclinical models: a protocol for a novel application of network meta-analysis. F1000Research. 2019;8:11-26. doi:10.12688/ f1000research.15869.1

6. Sun D, Wang W, Wang X, et al. bFGF plays an europrotective role by suppressing excessive autophagy and apoptosis after transient global cerebral ischemia in rats. Cell Death Dis. 2018;9:172. doi:10.1038/ s41419-017-0229-7

7. Miao Y, Liao JK. Potential serum biomarkers in the pathophysiological processes of stroke. Expert Rev Neurother. 2014;2:173-185. doi:10.1586/14737175.2014.875471

8. Zhou YP, Li GC. Kaempferol protects cell damage in in vitro ischemia reperfusion model in rat neuronal PC12 cells. Biomed Res Int. 2020;23:2461079.

9. Xu W, Zheng J, Gao L, Li T, Zhang J, Shao A. Neuroprotective effects of stem cells in ischemic stroke. Stem Cells Int. 2017;2017:4653936. doi:10.1155/2017/4653936 
10. Liang Y, Xu J, Wang Y, et al. Inhibition of miRNA-125b decreases cerebral ischemia/reperfusion injury by targeting $\mathrm{CK} 2 \alpha / \mathrm{NADPH}$ oxidase signaling. Cell Physiol Biochem. 2018;45:1818-1826. doi:10.1159/000487873

11. Del Zoppo GJ, Saver JL, Jauch EC, Adams HP Jr Expansion of the time window for treatment of acute ischemic stroke with intravenous tissue plasminogen activator: a science advisory from the American Heart Association/American Stroke Association. Stroke. 2009;40:2945-2948. doi:10.1161/STROKEAHA.109.192535

12. Sandercock P, Wardlaw JM, Lindleyetal RI, Dennis M, Cohen G, Murray G. The benefits and harms of intravenous thrombolysis with recombinant tissue plasminogen activator within $6 \mathrm{~h}$ of acute ischaemic stroke (The Third International Stroke Trial [IST-3]): a randomised controlled trial. Lancet. 2012;379:2352-2363.

13. Zhang Y, Wang YT, Luo JX, et al. Effect of WSP, a Chinese Herbal Formula, on Th17/Treg ratio and HBeAg seroconversion in telbivudine-treated HBeAg-positive chronic Hepatitis B patients with high baseline ALT levels (20-30 times the ULN). Evid Based Complement Alternat Med. 2019;2019:7260369.

14. Man MQ, Hu LZ, Elias PM. Herbal medicines prevent the development of atopic dermatitis by multiple mechanisms. Chin J Integr Med. 2019;25:151-160. doi:10.1007/s11655-015-2438-1

15. Hang YR, Liu CL, Jia K. The clinical efficacy of xiaoyaosan combined with dairenxin in the treatment of post-stroke depression and its effect on neurotransmitter levels. Inf Tradit Chin Med. 2019;36:84-87.

16. Wang JY. Effect analysis of xiaoyaosan in the treatment of 68 patients with post-stroke depression. A Clu Con Med Theo. 2019;17:206-208.

17. Sun QY. A randomized, parallel controlled study of xiaoyao SAN combined with fluoxetine in the treatment of post-stroke depression. J Inter Med Appl Tradit Chin Med. 2017;31:66-67.

18. Yang X, Su HP, Yang HF. Clinical efficacy and mechanism of xiaoyaosan combined with sertraline in patients with post-stroke depression. World Tradit Chin Med. 2018;13:60-63.

19. Wang C, Wu C, Yan ZG, Cheng X. Ameliorative effect of Xiaoyaojieyu-san on post-stroke depression and its potential mechanisms. J Nat Med. 2019;73:76-84. doi:10.1007/s11418-018-1243-5

20. Shi BY, Liu R, Rao ZL, et al. Protective mechanism of xiaoyaosan on nerve injury induced by LPS in rats. Chin $J$ Exp Form. 2019;025:50-56.

21. Wang MM, Bi YM, Zeng SM, et al. Modified Xiaoyao San ameliorates depressive-like behaviors by triggering autophagosome formation to alleviate neuronal apoptosis. Biomed Pharmacother. 2019;111:1057-1065. doi:10.1016/j.biopha.2018.12.141

22. Peng W, Shen H, Lin B, et al. Docking study and antiosteoporosis effects of a dibenzylbutane lignan isolated from Litsea cubeba targeting cathepsin K and MEK1. Med Chem Res. 2018;27:2062-2070. doi:10.1007/s00044-018-2215-8

23. Zheng TT, Shi Y, Zhang J, et al. MiR-130a exerts neuroprotective effects against ischemic stroke through PTEN/PI3K/AKT pathway. Biomed Pharmacother. 2019;117:109117. doi:10.1016/j. biopha.2019.109117

24. Wang SM, Long SQ, Xiao SJ, Wu WY, Hann SS. Decoction of Chinese Herbal Medicine Fuzheng Kang-Ai Induces lung cancer cell apoptosis via STAT3/Bcl-2/Caspase-3 pathway. Evid Based Complement Alternat Med. 2018;2018:1-14.
25. Wang YE, Xu K, Yue WH, et al. Hederacolchiside A1 suppresses proliferation of tumor cells by inducing apoptosis through modulating PI3K/Akt/mTOR signaling pathway. Chin Herbal Med. 2018: S1674638418300327.

26. Zhang Q, Peng W, Wei SJ, et al. Guizhi-Shaoyao-Zhimu decoction possesses anti-arthritic effects on type II collagen-induced arthritis in rats via suppression of inflammatory reactions, inhibition of invasion \& migration and induction of apoptosis in synovial fibroblasts. Biomed Pharmacother 2019;118:109367. doi:10.1016/j.biopha.2019.109367

27. Wu F, Qiu J, Fan Y, et al. Apelin-13 attenuates ER stress-mediated neuronal apoptosis by activating $\mathrm{G} \alpha \mathrm{i} / \mathrm{G} \alpha \mathrm{q}-\mathrm{CK} 2$ signaling in ischemic stroke. Exp Neurol. 2018;302:136-144. doi:10.1016/j.expneurol.2018.01.006

28. Uzdensky AB. Apoptosis regulation in the penumbra after ischemic stroke: expression of pro- and antiapoptotic proteins. Apoptosis. 2019;24(Suppl 2):687-702. doi:10.1007/s10495-019-01556-6

29. Kalpage HA, Bazylianska V, Recanati MA, et al. Tissue-specific regulation of cytochrome $\mathrm{c}$ by post-translational modifications: respiration, the mitochondrial membrane potential, ROS, and apoptosis. FASEB J. 2019;33:1540-1553. doi:10.1096/fj.201801417R

30. Peng W, Qiu XQ, Shu ZH, et al. Hepatoprotective activity of total iridoid glycosides isolated from Paederia scandens (lour.) Merr. var. tomentosa. J Ethnopharmacol. 2015;174:317-21. 5. doi:10.1016/j.jep.2015.08.032

31. Valko M, Leibfritz D, Moncol J, Cronin MTD, Mazur M, Telser J. Free radicals and antioxidants in normal physiological functions and human disease. Int J Biochem Cell Biol. 2007;39:44-84. doi:10.1016/ j.biocel.2006.07.001

32. Wang MQ, Hu R, Wang YJ, et al. Atractylenolide III attenuates muscle wasting in chronic kidney disease via the oxidative stress-mediated PI3K/AKT/mTOR pathway. Oxid Med Cell Longev. 2019:1875471.

33. Lei SQ, Su WT, Xia ZY, et al. Hyperglycemia-induced oxidative stress abrogates remifentanil preconditioning- mediated cardioprotection in diabetic rats by impairing caveolin-3Modulated PI3K/Akt and JAK2/STAT3 signaling. Oxid Med Cell Longev. 2019;2019:9836302. doi:10.1155/2019/9836302

34. Li HL, Tang ZY, Chu P, et al. Neuroprotective effect of phosphocreatine on oxidative stress and mitochondrial dysfunction induced apoptosis in vitro and in vivo: involvement of dual PI3K/Akt and Nrf2/HO-1 pathways. Free Radic Biol Med. 2018;120:228-238. doi:10.1016/j.freeradbiomed.2018.03.014

35. Zhang Q, Liu J, Zhang MM, et al. Apoptosis induction of fibroblast-like synoviocytes is an important molecular-mechanism for herbal medicine along with its active components in treating rheumatoid arthritis. Biomolecules. 2019;9:795. doi:10.3390/ biom 9120795

36. Zhang K, Tu MJ, Gao W, et al. Hollow prussian blue nanozymes drive neuroprotection against ischemic stroke via attenuating oxidative stress, counteracting inflammation, and suppressing cell apoptosis. Nano Lett. 2019;19:2812-2823. doi:10.1021/acs. nanolett.8b04729

\section{Publish your work in this journal}

Drug Design, Development and Therapy is an international, peerreviewed open-access journal that spans the spectrum of drug design and development through to clinical applications. Clinical outcomes, patient safety, and programs for the development and effective, safe, and sustained use of medicines are a feature of the journal, which has also been accepted for indexing on PubMed Central. The manuscript management system is completely online and includes a very quick and fair peer-review system, which is all easy to use. Visit http://www. dovepress.com/testimonials.php to read real quotes from published authors. 